\title{
Exhaustive Search-based Model for Hybrid Sensor Network
}

\author{
A.A. Waskita*†, H. Suhartanto ${ }^{\dagger}$, Z. Akbar ${ }^{\ddagger \S}$, L.T. Handoko A $^{\ddagger}$ \\ *Center for Development of Nuclear Informatics, National Nuclear Energy Agency, \\ Kawasan Puspiptek Serpong, Tangerang 15310, Indonesia \\ Email : adhyaksa@batan.go.id \\ ${ }^{\dagger}$ Faculty of Computer Science, University of Indonesia, \\ Kampus UI Depok, Depok 16424, Indonesia \\ Email : heru@cs.ui.ac.id \\ ${ }^{\ddagger}$ Group for Theoretical and Computational Physics, Research Center for Physics, Indonesian Institute of Sciences, \\ Kawasan Puspiptek Serpong, Tangerang 15310, Indonesia \\ Email: zaenal@teori.fisika.lipi.go.id, handoko@teori.fisika.lipi.go.id

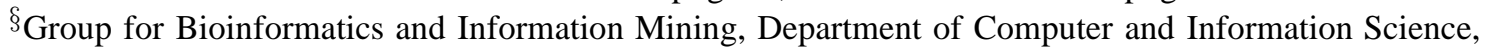 \\ University of Konstanz, Box D188, D-78457 Konstanz, Germany \\ Email: zaenal.akbar@uni-konstanz.de \\ TDepartment of Physics, University of Indonesia, \\ Kampus UI Depok, Depok 16424, Indonesia \\ Email: handoko@fisika.ui.ac.id
}

\begin{abstract}
A new model for a cluster of hybrid sensors network with multi sub-clusters is proposed. The model is in particular relevant to the early warning system in a large scale monitoring system in, for example, a nuclear power plant. It mainly addresses to a safety critical system which requires real-time processes with high accuracy. The mathematical model is based on the extended conventional search algorithm with certain interactions among the nearest neighborhood of sensors. It is argued that the model could realize a highly accurate decision support system with less number of parameters. A case of one dimensional interaction function is discussed, and a simple algorithm for the model is also given.
\end{abstract}

\section{INTRODUCTION}

A safety critical system in, for example, nuclear power plants involves complicated and advance safety system for ensuring its running process absolutely safe. The system consists of high number and various types of sensors [1], [2]. All of them generate a huge amount of data at a real-time basis which must be processed properly throughout its whole life time. Many heuristic approaches based on the artificial intelligence (AI) such as neural network are currently available and have been studied intensively [3]-[6]. However, the AI-based approaches have a fundamental problem due to its statistical algorithm which could lead to disaster in the real applications of safety critical system.

It is clear that in such critical systems, no fault tolerance is the most important principle. Therefore, putting the safety as the priority, one should implement the exhaustive algorithm spanning over all possibilities rather than using the AIs. This turns into the exhaustive search problem which unfortunately lacks of inefficiency due to the requirement of huge computing power. Some technical approaches have been introduced to overcome this problem. Most of them deploy the parallel algorithm [7], [8] together with graphical or combinatorial representation to improve both resources and running time [9][12].

Previously, the application of exhaustive methods into search problem was not feasible for large number of sensors. Fortunately, the affordable parallel environment using graphic processor unit (GPU) is available in recent days. The use of GPU is getting popular, especially, after the introduction of the NVIDIA Compute Unied Device Architecture (CUDA) through a C-based API [13]. This enables an easy way to take advantage of the high performance of GPUs for parallel computing. Deploying the GPU-based distributed computing would reduce the execution time causing less responsive system in the previous days, while it also realizes lower power and space consumption than CPU [14]. This motivates us to reconsider the feasibility of exhaustive search for hybrid sensors network.

In this paper a new exhaustive search based model is proposed. The model is mainly intended to realize an exhaustive decision support system (DSS) consisting of various and huge number of sensors. However, the paper is focused only on introducing the model. The discussion of parallelization and detail analysis will be published elsewhere.

The paper is organized as follows. First in Sec. II the model is introduced, and it is followed by the description of mathematical formulation in Sec. III. In Sec. IV a simple algorithm to execute the model is given. Finally, the paper is ended with a short summary and discussion.

\section{THE MODEL}

Before moving on constructing the model and its mathematical representation, let us discuss the basic constraints and 
circumstances in the expected applications. Putting in mind that the model is developed under the following considerations :

1) No failure decision is allowed.

By definition, there is no room for even a small mistake generated by the DSS. This actually discourages the deployment of any AI-based method from first principle.

2) Fast enough 'real-time' process

Fast execution time of the whole process is crucial to increase the safety. However, fast execution time at the order of minutes is in practical more than enough. This moderate requirement encourages the implementation of exhaustive methods supported by GPU powered computation.

3) Huge number of hybrid sensors

The system is consisting of huge number (at the order of hundreds or thousands) sensors with different characteristics, in particular types and scales [15].

4) Certain relationship across the sensors

Each sensor has relations with another ones in certain way at some degrees. The relationships among the sensors are thereafter called as interaction.

\section{5) Sensor network with multi clusters}

The sensor network is divided into several clusters which typically represents the geographical locations with different degrees of interaction. This realizes a situation of, for instance, a nuclear power plant which is equipped with many sensors in each building. Consequently the sensors in each cluster have stronger interactions among themselves than with another ones belong to another clusters. So, the model should be able to describe the independent interactions among the sensors in a cluster, and also the interactions among different clusters as well.

6) Dynamic behavior

The values of each sensor are by nature changing from time to time. However, the data acquisition is performed periodically, for instance every few minutes according to the above second point. In a nuclear reactor facility this could happen due to human errors, common system failures and even seismic activities [16].

Having the above requirements in mind, obviously one will arrive at the problem of unlimited decision trees. In order to reduce the tree significantly without raising the risk of failures, let us assume the nearest neighborhood approximation (NNA). Under this approximation, only the interactions with the nearest sensors are taken into account.

In the present paper, let us consider the simplest case of one dimensional (1-D) relationship. This means all sensors are put on a virtual line which allows only interactions with the nearest right and left neighboring sensors for each sensor. This actually reproduces the known tree analysis commonly implemented in the analysis of fault [16], [17], elements interaction [18]-[20] and even in optimizing system [21].

The algorithm can be well illustrated in a tree-like diagram using the evaluation scheme under the NNA scheme as shown in Fig. 1. In the figure, two adjacent sensors are first evaluated

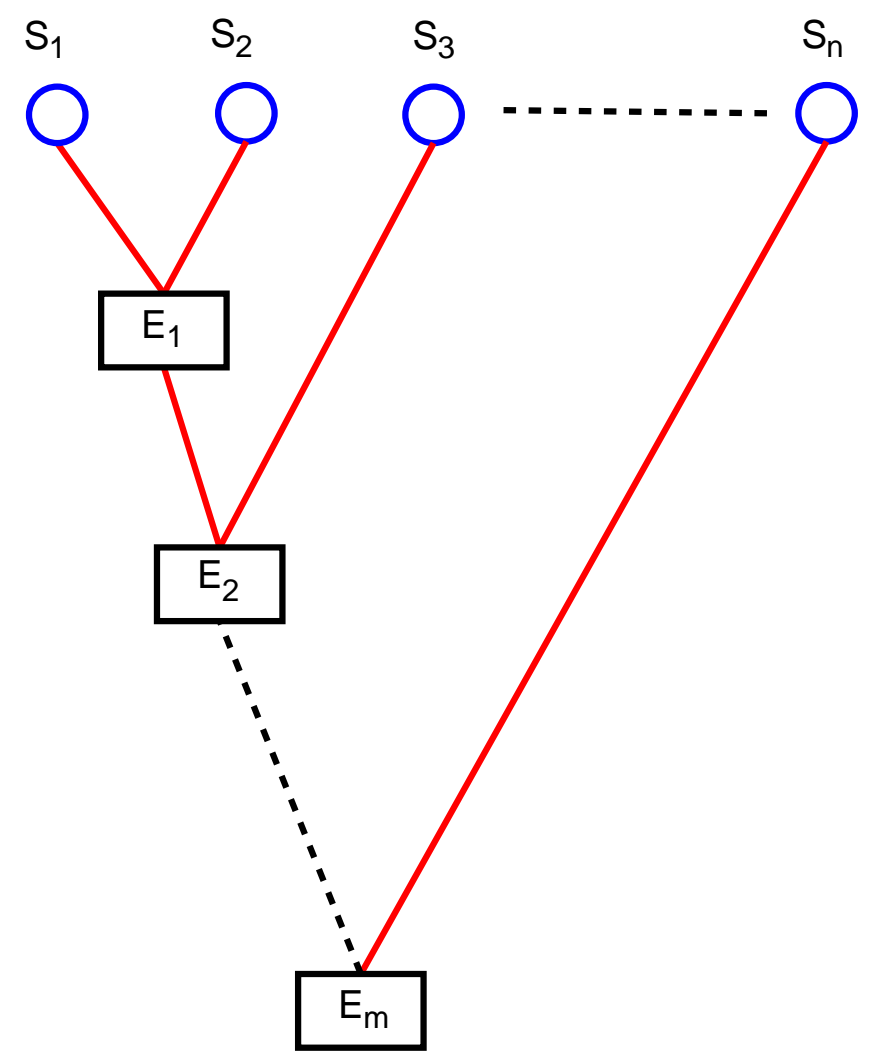

Fig. 1: The illustration of 1-D relationship of $n$ sensors evaluated till the $m^{\text {th }}$ level, where $S$ denotes sensor and $E$ is the evaluation result.

and then the result are subsequently evaluated with another adjacent sensor. The evaluation scheme depicted in Fig. 1 can be exhaustively changed according to the acquired values from the responsible sensors. It should be noted that the evaluation scheme is not necessarily binary, but it could be anything else like fuzzy and so on.

On the other, due to point 4 one should consider the modified tree analysis, that is both edges should interact each other too and forms a circle line of sensors. Moreover, each sensor on the circle line should be put carefully. Because, according to point 5 and the NNA, the relative location of sensors on the circle line represents their degree of relationship or relevancy between one and another. The stronger relationship between two sensors, both should be put closer each other. This type of circular model is depicted in Fig. 2. There are two examples of evaluation results in the figure, the blue and brown ones corresponding to the evaluation at different time. The innermost circles represent the chain of sensors, and the subsequent outer circles describe the evaluation results at certain levels.

As required in point 5, the sensor network should also be divided into several clusters based on either its genuine characteristic or critical levels. Each cluster can be treated separately as an independent sensor network as Fig. 2. The model with several clusters is illustrated in Fig. 3 where each cluster is separated by the blue dashed lines. Two evaluation 


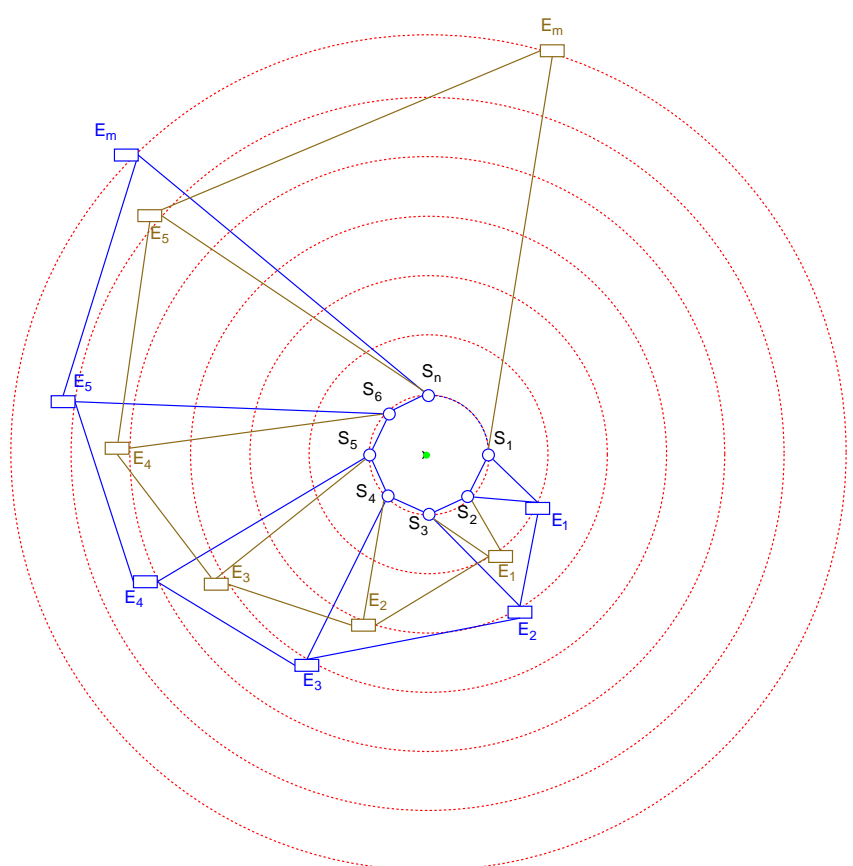

Fig. 2: The circular representation for 1-D relationship of $n$ sensors evaluated for two schemes (blue and brown lines) till the $m^{\text {th }}$ level, where $S$ denotes sensor and $E$ is the evaluation result.

results are shown in the figure as before, the blue and brown ones corresponding to the evaluation at different time.

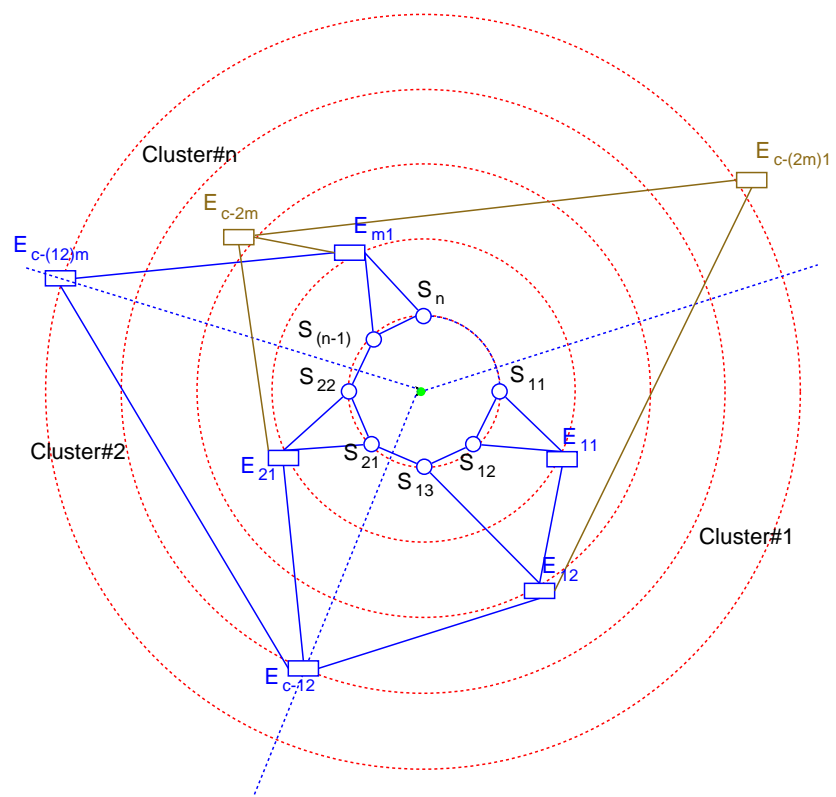

Fig. 3: The circular representation for 1-D relationship of $n$ sensors belong to separated clusters, evaluated for two schemes (blue and brown lines) till the $m^{\text {th }}$ level, where $S$ denotes sensor and $E$ is the evaluation result.
TABLE I: The values of weight parameter $a_{i j}$ for $n$ sensors.

\begin{tabular}{c|cccccc}
$a_{i j}$ & 1 & 2 & 3 & $\cdots$ & $n-1$ & $n$ \\
\hline 1 & 1 & $\frac{n-1}{n}$ & $\frac{n-2}{n}$ & $\ldots$ & $\frac{2}{n}$ & $\frac{1}{n}$ \\
2 & $\frac{n-1}{n}$ & 1 & $\frac{n-1}{n}$ & $\ldots$ & $\frac{3}{n}$ & $\frac{2}{n}$ \\
3 & $\frac{n-2}{n}$ & $\frac{n-1}{n}$ & 1 & $\ldots$ & $\frac{4}{n}$ & $\frac{3}{n}$ \\
$\vdots$ & $\vdots$ & $\vdots$ & $\vdots$ & 1 & $\vdots$ & $\vdots$ \\
$n-1$ & $\frac{2}{n}$ & $\frac{3}{n}$ & $\frac{4}{n}$ & $\ldots$ & 1 & $\frac{n-1}{n}$ \\
$n$ & $\frac{1}{n}$ & $\frac{2}{n}$ & $\frac{3}{n}$ & $\cdots$ & $\frac{n-1}{n}$ & 1
\end{tabular}

Now, one is ready to formulate the model in a mathematical representation.

\section{MATHEMATICAL REPRESENTATION}

Based on the previous discussion, one should first model the interaction between the neighboring sensors. This should be a function which determines the value of $E_{i, j}$ representing the interacting result between two adjacent sensors at the point $i$ and $j$,

$$
E_{i j}=a_{i j}\left(\frac{x_{i}}{l_{i}+1}+\frac{x_{j}}{l_{j}+1}\right) .
$$

Here, $l_{i}=1,2, \cdots, m$ denotes the evaluation level of $x_{i}$ with $i=1,2, \cdots, n$ and $m=n-1$. While $x_{i}$ is the normalized value acquired by sensor for $l_{i}=1$, or the value of previous evaluation result for $l_{i}>1$. The coupling constant $a_{i j}$ reflects the strength of interaction between both adjacent sensors, $x_{i}$ and $x_{j}$ respectively. It is defined in a way such that the value of $E_{i j}$ is getting smaller for higher evaluation level, that is,

$$
a_{i j}=1-\frac{|i-j|}{n},
$$

for $l_{i}=1$ in a system with $n$ sensors.

Moreover, Eqs. (1) and (2) can be extended for $l_{i}>1$ as follow,

$$
E_{(i j) k}=a_{i j k}\left(\frac{x_{i j}}{l_{i j}+1}+\frac{x_{k}}{l_{k}+1}\right),
$$

with,

$$
a_{i j k}=\frac{a_{i j}+a_{i k}+a_{j k}}{3},
$$

and $x_{i j}=E_{i j}$ respectively. The situation is well illustrated in Figs. 2] and 3. Further generalization of Eqs. (3) and (4) up to certain evaluation level can be performed in a straightforward way.

There is actually an important reason for choosing the definition in Eqs. (2) and (4). In order to fulfill the requirements in point 3 and 4 in Sec. II it is plausible to normalize all scales in a uniform unit scale. In the present case all values are normalized to be in the range between 0 and 1. This normalization demands all acquired values from the sensors should also be normalized accordingly through a relation,

$$
x_{i}=f\left(x_{i}^{\prime}-x_{\min }\right),
$$


where $x_{i}$ and $x_{i}^{\prime}$ are the normalized and originally acquired values of $i^{\text {th }}$ sensor for $l_{i}=1$. The normalization factor $f$ is,

$$
f=\frac{1}{\left|x_{\max }-x_{\min }\right|},
$$

with $x_{\max / \min }$ denotes the maximum or minimum value of each sensor. This kind of normalization enable us to treat all sensors in the same manner regardless with its types and unit scales.

From Eqs. (2) and (4), it is obvious that the coupling $a$ is uniquely characterizing the present model. It ensures the evaluation value of $E$ at the final $(n-1)^{\text {th }}$ level is always divergent, that is between 0 and 1 . This is in contradiction with any conventional tree analysis which associates the largest evaluation value at the final level as the final solution. By the way, from the definition $1 / n \leq a_{i j} \leq 1$ as shown in Tab. I which forms a symmetric matrix with unit diagonal elements.

Furthermore, one should take a threshold value $E_{\text {th }}$ as a standard value whether the evaluation value at certain level is allowed to proceed further or not. For the sake of simplicity, this value is fixed and valid for all levels and sensors. This represents the critical value of safety. Following the above normalization it is again constrained,

$$
0<E_{\text {th }}<1 .
$$

Only if the evaluation value exceeds this threshold, i.e. $E>$ $E_{\mathrm{th}}$, the tree should be analyzed further. Otherwise it ends forever. According to the initial value of each sensor at certain time, some evaluation values at final level may survive or not. The surviving value triggers the warning alarm which is indicating some anomalies detected by any sensors. Of course, the determination of appropriate $E_{\text {th }}$ requires preliminary experiments based on the available standards and regulations.

The above procedure is carried out each time following the periodic data acquisition by all sensors. Finally, all the tools have been established and we are ready for applying the above rules.

\section{THE ALGORITHM}

In this section, let us provide a simple algorithm to realize the previously discussed model.

Because of the exhaustive method deployed in the model, it requires all variables are treated as a circle. The array of variables should be a traversing pointer indicating the first element as a starting point. Further, the evaluation is performed from the starting point, proceeds to the subsequent element of variables array in an increasing mode till reaching the last one, that is back to the first element. Each sensor has a chance to become a root of a tree and also a leaf.

While forming a circle model, the tree within the model is evaluated recursively. A set of simple algorithms is presented here. It consists of two main parts for the pra-evaluation and the main evaluation. Each sensor is labeled with an integer ranging from 0 to $n-1$ for $n$ number of sensors. The algorithm starts with positioning the sensors by shifting it one by one to generate considerable combinations. Each time a tree of sensors is formed, it is evaluated as a scheme depicted in Fig. 1.
Algorithm 1,3 require the array of sensor's indexes and values. The value described in a sensor's index array absolutely points to its value in the array of sensor's value. Algorithm 1 determines the positioning of sensors, while Algorithm 2 and 3 evaluate their interactions.
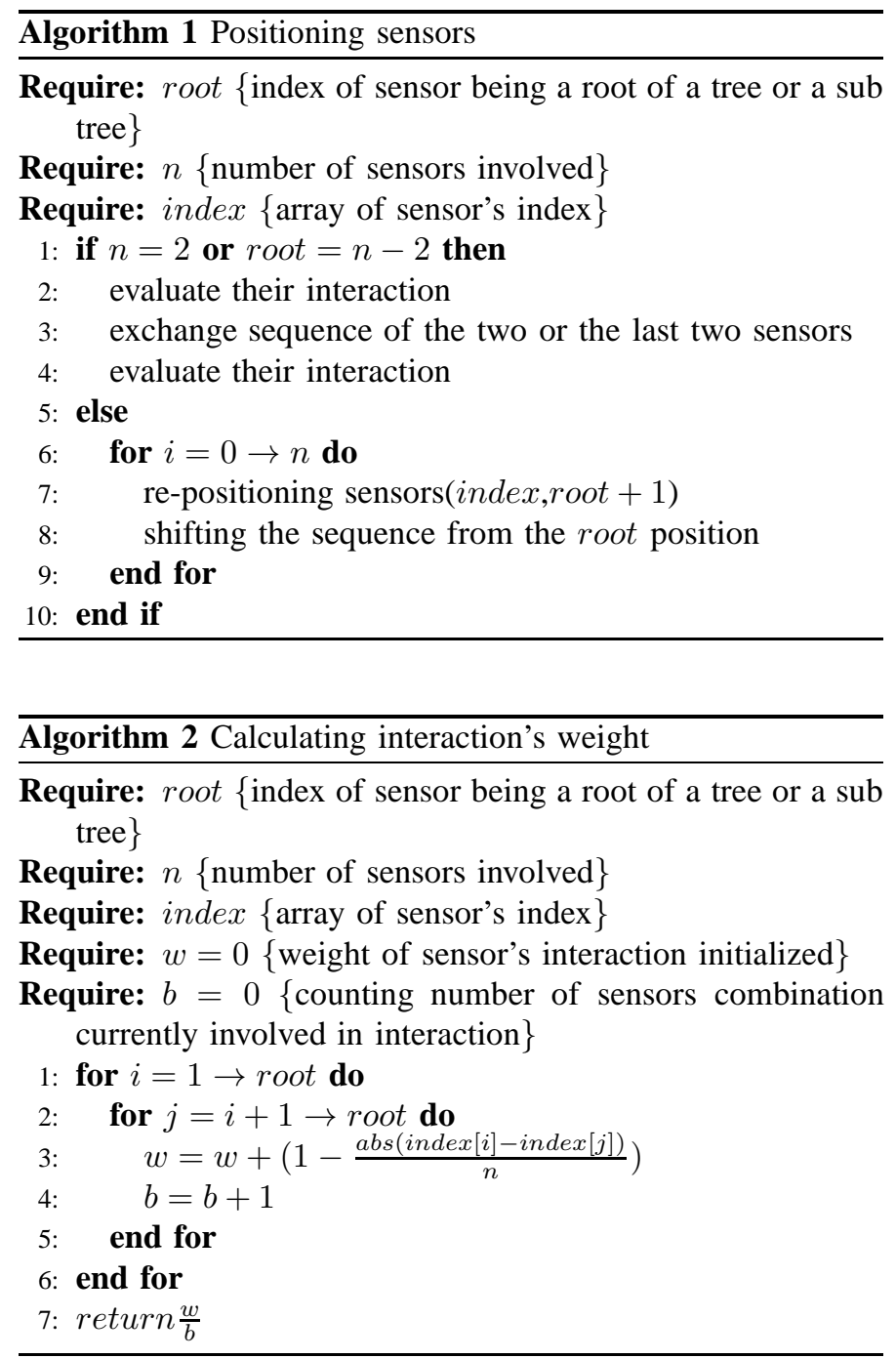

In positioning the sensors as determined by Algorithm 1 , for the case of two sensors the algorithm only exchanges their sequences. After the evaluation of first sequence, the algorithm exchanges the sequence and reevaluate the new one. This procedure is specified in the second to fourth lines. For the case of more than two sensors, the algorithm recursively traverses 
the sequence till it reaches the condition where only two sensors are left. In this case, the algorithm runs in the same manner as if there are only two sensors involved. Each time the algorithm is traversing deeper, the root is increased indicating the depth of tree under evaluation. If a leaf of tree is reached, the algorithm returns back to the parent leaf, exchanges to the next root, traverses deeper till it reaches the leaf. After all paths to each leaf have been reached by one root, the sequence exchanges to another sensor as the new root and so forth.

Furthermore, the weight of interaction is calculated using Eq. (2) and given in Tab. II In Algorithm 1 root determines the root of a new sub tree, while in Algorithm 2 root is intended to determine the number of sensors which are currently involved in the interaction as illustrated in Fig. 1, $b$ in Algorithm 2 is intended to count the number of combination of two sensors among the whole sensors as defined in Eq. 4, that is it would be as many as elements in Tab. I. For example, the interaction of three sensors contains three dual-sensor interactions, while the interaction of four sensors contains six and so on.

Finally, Algorithm 3 is intended to calculate the evaluation value, $E$. It requires the weight of each interaction from Algorithm 2 using Eq. (2) and Tab. I. The total number of evaluation values is equal to the number of sensors being involved.

\section{SUMmary}

A new model based on the exhaustive search method for hybrid sensor network has been proposed. The model treats all sensors in the same manner by introducing normalization procedure for all sensors and parameters. It is shown that the model is able to describe the whole evaluation processes using few parameters, that is the coupling constant $a$ for each pair of sensors determined uniformly and the universal threshold value $E_{\text {th. }}$.

In the present paper, the study is focused on the case of sensor network with 1-D relationship. A simple algorithm for such cases has also been given and briefly discussed. Through the discussion, it is argued that the model could realize a feasible early warning system for any safety critical facilities involving various sensors using exhaustive method to prevent unnecessary failures due to statistical approaches in, for example any AI-based methods. On the other hand, the method requires much less computing power since it has only a complexity of $O(n !)$.

In principle the method can be extended to incorporate more complicated relationship among the sensors by considering higher dimensional relationship. Nevertheless, some studies on distributing the computation load to improve the processing speed should also been done carefully. All of these issues are in progress and will be published elsewhere.

\section{ACKNOWLEDGMENT}

AAW thanks the Indonesian Ministry of Research and Technology for financial support, and appreciates the Group for Theoretical and Computational Physics at Research Center for Physics LIPI for warm hospitality during the work. The work of LTH is supported by the Riset Kompetitif LIPI 2012 under Contract no. 11.04/SK/KPPI/II/2012.

\section{REFERENCES}

[1] J. Affandi and E. Rosadi, "Identification of Faults in A Reactor Coolant Pump (RCP) System," in Risalah Lokakarya Komputasi dalam Sains dan Teknologi Nuklir XII, 2001, pp. 305-322.

[2] H. M. Hashemian, "Process sensors for nuclear power plants," $2010 . \quad$ [Online]. Available: http://www.scitopics.com/Process_Sensors_for_Nuclear_Power_Plants.html

[3] I. Obreja, "Diagnosis of power plant faults using qualitative models and heuristic rules," in 3rd international conference on Industrial and engineering applications of artificial intelligence and expert systems Volume 1, vol. 1, 1990, pp. 41-46.

[4] T. Jayasree, D. Devaraj, and R. Sukanesh, "Classification of transients using wavelet based entropy and radial basis neural networks," International Journal of Computer and Electrical Engineering, vol. 1, no. 5, pp. 590-595, 2009.

[5] E. Zio, M. Broggi, and N. Pedroni, "Nuclear reactor dynamics on-line estimation by locally recurrent neural networks," Progress in Nuclear Energy, vol. 51, pp. 573-581, 2009.

[6] D. Sutarya and B. Kusumoputro, "Quality classification of uranium dioxide pellets for pwr reactor using anfis," in Proceeding of IEEE Technical Conference 2011, 2011, pp. 314-318.

[7] E. D. Karnin, "A Parallel Algorithm for the Knapsack Problem," IEEE Transactions on Computers, vol. C-33, no. 5, pp. 404-408, 1984.

[8] L. Hui and C. Yonghui, "Study of heuristic search and exhaustive search in search algorithms of the structural learning," in 2nd International Conference on Multimedia and Information Technology, vol. 1, 2010, pp. 169-171.

[9] S. Matwin and T. Pietrzykowski, "Intelligent Backtracking in PlanBased Deduction," IEEE Transactions on Pattern Analysis and Machine Intelligence, vol. PAMI-7, no. 6, pp. 682-692, 1985.

[10] J. Nievergelt, R. Gasser, F. Mäser, and C. Wirth, "All the needles in a haystack: Can exhaustive search overcome combinatorial chaos?" in Lecture Notes in Computer Science : Computer Science Today. Springer, 1995, pp. 254-274.

[11] P. Bhalchandra, N. Deshmukh, R. Rathod, S. Khamitkar, S. Lokhande, and S. Phulari, "New perspective for tree searching algorithms: A simple proposition," in 2nd International Conference on Computer Technology and Development, 2010, pp. 10-16.

[12] R. Y. Chang and W.-H. Chung, "Efficient Tree-Search MIMO Detection with Probabilistic Node Ordering," in IEEE International Conference on Communications, june 2011, pp. 1-5.

[13] "What is CUDA ?" 2011. [Online]. Available: http://developer.nvidia.com/what-cuda

[14] V. Boyer, D. E. Baza, and M. Elkihel, "Solving knapsack problems on gpu," Computers and Operations Research, vol. 39, pp. 42-47, 2012.

[15] S. Yoon, J. Jo, and J. Yoo, "A domain-specific safety analysis for digital nuclear plant protection systems," in 5th International Conference on Secure Software Integration Reliability Improvement Companion (SSIRIC), 2011, june 2011, pp. 68-75.

[16] O. P. M. NUSBAUMER, "Analytical solutions of linked fault tree probabilistic risk assessments using binary decision diagramswith emphasis on nuclear safety applications," Ph.D. dissertation, SWISS FEDERAL INSTITUTE OF TECHNOLOGY ZURICH, 2007.

[17] Q. Geng, H. Duan, and S. Li, "Dynamic fault tree analysis approach to safety analysis of civil aircraft," in 6th IEEE Conference on Industrial Electronics and Applications (ICIEA), 2011, june 2011, pp. 1443-1448.

[18] B. hoon Park, M. Schmidt, K. Thomas, T. Karpinets, and N. F. Samatova, "Parallel, scalable, memory-efficient backtracking for combinatorial modeling of large-scale biological systems," Proceeding of IEEE International Symposium on Parallel and Distributed Processing, 2008. IPDPS 2008., pp. 1-8, 2008.

[19] W. Hendrix, M. C. Schmidt, P. Breimyer, and N. F. Samatova, "On perturbation theory and an algorithm for maximal clique enumeration in uncertain and noisy graphs," in Proceedings of the 1st ACM SIGKDD Workshop on Knowledge Discovery from Uncertain Data. ACM, 2009, pp. 48-56.

[20] C. Lee, F. Reid, A. McDaid, and N. Hurley, "Detecting highly overlapping community structure by greedy clique expansion," in Proceeding of The 4th SNA-KDD Workshop, 2010. 
[21] C. Jiang, L. Bai, and W. Zheng, "Research on layout of airport logistics park based on graph theory: An empirical study of ningbo airport logistics park," in Proceeding of International Conference on Intelligent Computation Technology and Automation (ICICTA), 2010. 